Research Article

\title{
Detection of Fatigue Microcrack Using Eddy Current Pulsed Thermography
}

\author{
Xiang Zhang $\mathbb{D},{ }^{1}$ Jianping Peng $\mathbb{D},{ }^{1}$ Luquan Du, ${ }^{2}$ Jie Bai, ${ }^{1}$ Lingfan Feng, ${ }^{1}$ Jianqiang Guo, ${ }^{1}$ \\ and Xiaorong Gao ${ }^{1}$ \\ ${ }^{1}$ School of Physical Science and Technology, Southwest Jiaotong University, Chengdu 610031, China \\ ${ }^{2}$ School of Electronic Information and Automation, ABA Teachers University, ABA, 623002, China \\ Correspondence should be addressed to Jianping Peng; adams.peng@swjtu.edu.cn
}

Received 16 December 2020; Revised 16 January 2021; Accepted 17 March 2021; Published 7 April 2021

Academic Editor: Bin Gao

Copyright (c) 2021 Xiang Zhang et al. This is an open access article distributed under the Creative Commons Attribution License, which permits unrestricted use, distribution, and reproduction in any medium, provided the original work is properly cited.

\begin{abstract}
Microcracks are a common metallic defect, resulting in degradation of material properties. In this paper, specimens with different fatigue microcracks were detected by eddy current pulsed thermography (ECPT). Signal processing algorithms were investigated to improve the detectability and sensitivity; principal component analysis (PCA) and Tucker decomposition were used to compare the performance of microcrack detection. It was found that both algorithms were highly adaptable. A thermal quotient was used to assess the temperature variation trend. Furthermore, the potential correspondence between crack closure and temperature change was investigated.
\end{abstract}

\section{Introduction}

In recent years, infrared (IR) technology has been successfully applied to power, rail, and other fields because of its advantages for noncontact, high sensitivity, and visualization [1-4]. As active infrared thermography, eddy current pulsed thermography (ECPT) uses external high-frequency alternating current to excite the detection coil so as to generate heat on the surface or the inside of the measured specimen [5].

In-service equipment may have complex natural defects due to stress and impact loads [6]. Several results based on ECPT in the fields of crack detection have been reported. Specifically, Vrana et al. [7] proposed simplified models for crack detection with induction thermography. Shi et al. [8] proposed a quantitative crack detection method, and cracks of different sizes were analysed. Weekes et al. [9] performed an experimental investigation of fatigue cracks in steel, titanium, and nickel-based superalloy, and results of probability of detection (POD) were established. Tong et al. [10] modified the modelling of ECPT to achieve quantitative evaluation of blade surface fatigue cracks in heavy-duty gas turbines. Netzelmann et al. [11] employed the ECPT method demonstrating the detection of hardening cracks on a large gear tooth. Peng et al. [12] developed solutions for four types of winding defects; the defects under layer insulation can be detected using ECPT. Genest and Li [13] used both experimental and numerical assessments of the induction thermography technique, detecting the microcrack in notched steel coupons.

The abundant transient information in the ECPT has provided grounds for further analysis. In order to enhance the resolution, Maldague and Marinetti [14] proposed pulse phase thermography (PPT), which has an advantage in quantitative inversion. Chen et al. [15] used ICA to identify defect patterns automatically and reduced the influence of the emissivity. Wang et al. [16] used PCA to process the artificial crack in a steel sample and natural fatigue cracks in aircraft brake components. Zhang et al. [17] performed PCA and partial least-squares thermography (PLST) to improve the infrared image performance for defect detection in composite panels. Among the above dimension reduction and matrix decomposition methods, these algorithms provided manifest high levels of flaw contrast relative to that present in the unprocessed data. In addition, tensor decomposition approaches have become an effective tool for feature extraction in infrared thermography crack detection. Gao et al. 
[18] developed a spatial transient phase tensor model to extract and separate patterns. Song et al. [19] formulated a tensor decomposition analytical model to identify cracks on samples with different geometry.

However, previous research has rarely discussed the diagnosis of crack closure effect, which is common in the industry. In fatigue tests, it can typically be classified into three categories: plasticity-induced [20, 21], roughness-induced [22], and oxide-induced [23, 24] crack closures. Moreover, Jomdecha et al. [25] proposed a new model to calculate the magnetic flux density over the stress-corrosion crack (SCC) region of different conductivity. Chen et al. [26] found that SCC behaves like a conductive slit in the perspective of eddy current testing. Nevertheless, there remain challenges to distinguish the weak thermal features of closed cracks. In this study, we aim to examine applications of ECPT in fatigue microcrack in a more comprehensive way. Experimental tests on fatigue precrack by three-point bending have been conducted, which is an important step from artificial crack to natural fatigue crack. Compared with PCA, tensor decomposition could preserve more defect information. The performance of detecting fatigue crack is discussed through SNR and thermal quotient. The remainder of this paper is organized as follows: the methodology used in this work is described in Section 2. The experimental setup and specimen are explained in Section 3. The result and discussion are then provided in Section 4. Finally, the conclusion is outlined in Section 5 .

\section{Methodology}

2.1. Induction Heating Theory of ECPT. The main physical process of ECPT involves induced eddy current heating and thermal diffusion. These eddy currents are governed by a subsurface penetration depth, based on an exponentially damped skin effect. According to Joule's law, the thermal power generated by the internal resistance of the material is

$$
P_{w}=\frac{1}{\sigma}\left|J_{e}\right|^{2}=\frac{1}{\sigma}|\sigma E|^{2}
$$

where $J_{e}$ is the eddy current density and $E$ is the electric field strength; equation (1) determines the resulting temperature field. In general, the environmental temperature or reference temperature is taken as a constant $T_{0}$. Thus, the heat conduction equation of a specimen in the company of a defect can be expressed as

$$
\frac{\partial^{2} T}{\partial x^{2}}+\frac{\partial^{2} T}{\partial y^{2}}+\frac{\partial^{2} T}{\partial z^{2}}+\frac{1}{\lambda} q(x, y, z, t)=\frac{\rho C_{p}}{\lambda} \frac{\partial T}{\partial t},
$$

where $T=T(x, y, z, t)$ denotes the temperature distribution, $\lambda$ is the thermal conductivity of the material, $\rho$ is the density, and $C_{p}$ is the specific heat, and $q(x, y, z, t)$ is the internal heat generation function per unit volume and unit time.

2.2. Principal Component Analysis. For raw data, PCA is an unsupervised classification method; its strength is that it reduces the dimensionality of the data while keeping most

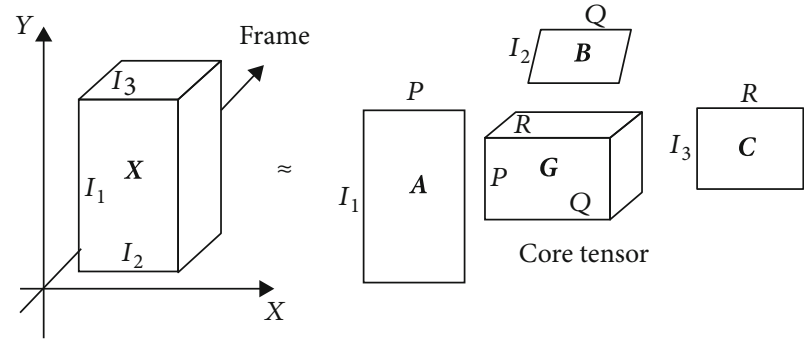

FIgURE 1: Decomposition of 3rd-order tensor.

of the variation in the data set. Each PC could be considered a linear combination of the original thermal sequences and ranked in decreasing order. Singular value decomposition and covariance matrix decomposition methods were used to decompose the 2-D thermal matrix in the following way:

$$
T=U R V^{T}
$$

where $U$ is a matrix; it contains a series of empirical orthogonal functions (EOFs). $R$ is a diagonal matrix with the singular values of $T ; V$ is an orthogonal matrix.

2.3. Tensor Decomposition. A tensor is a multidimensional array. The infrared sequences recorded by an IR camera can be represented by a third-order tensor $X \in R^{I_{1} \times I_{2} \times I_{3}}$, with two modes representing spatial position and the third mode representing the transient information. We decompose the higher-order tensor into a core tensor multiplied by a matrix along each mode. In the three-way case, the discretized tensor $X \in R^{I_{1} \times I_{2} \times I_{3}}$ can be calculated by Tucker decomposition as

$$
X \approx G \times{ }_{1} A \times{ }_{2} B \times{ }_{3} C=\sum_{p=1}^{P} \sum_{q=1}^{Q} \sum_{r=1}^{R} g_{p q r} a_{p} \circ b_{q} \circ c_{r},
$$

where $G \in R^{P \times Q \times R}$ is the core tensor; $A \in R^{I_{1} \times P}, B \in R^{I_{2} \times Q}$, and $C \in R^{I_{3} \times R}$ are the factor matrices considered as the principal components in each mode; and $P, Q$, and $R$ are the number of components in the factor matrices. The operator "o" denotes the vector's outer product. The tensor decomposition method does not perform dimensionality reduction on the thermal imaging high-dimensional image. Therefore, the tensor algorithm can maintain the structural stability of the original data and extract more crack features. This decomposition is illustrated in Figure 1.

2.4. Framework for This Work. Based on the theory introduction in this section, a research approach diagram for ECPT showing defect characterization in two specimens with different shapes was proposed, as shown in Figure 2. It was initiated by using an ECPT platform to acquire the raw data. Then, the thermal sequences in the region of interest (ROI) were preprocessed by PCA and tensor decomposition, respectively, to enhance the thermal contrast. After that, max thermal response and temperature line scan were used 


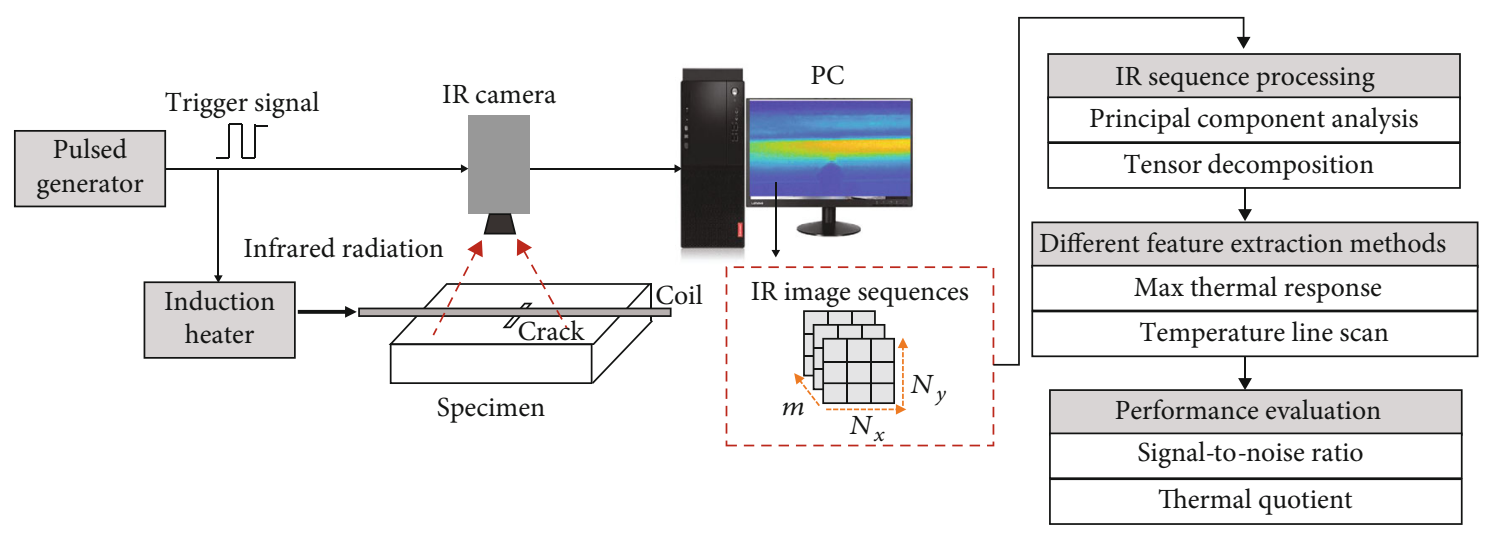

FIgURE 2: Inductive thermography and research approach diagram.

to extract the thermal features. Finally, the SNR and thermal quotient were used to evaluate the performance.

\section{Experiment Setup and Sample}

The rail specimen with machined notch (type $\mathrm{V}$ ) is described in Figure 3. Table 1 gives information about crack length, and Table 2 introduces the material properties. In this study, a straight-through notch of $1 \mathrm{~mm}$ in depth was introduced using spark discharge. The straight-through notch was placed connect with the machined notch's tip. Using this method, we were able to generate a well-defined and straight fatigue precrack using three-point bending. The setup of the three-point bending test is shown in Figure 3. Prior to producing the precrack, the specimen surfaces were polished with $\mathrm{SiC}$ paper up to $\# 1000$. After that, a straight fatigue crack was generated using a fatigue testing machine (MTS809, USA) with a sinusoidal $20 \mathrm{~Hz}$ waveform.

The specimen with precrack was then tested using the ECPT method with two conditions each before and after resection along the red dashed line in Figure 4 . That is, the specimens were machined into two types (after three-point bending tests) as illustrated in Figure 4, where $a$ is the fatigue precrack length, $c$ is the length of the machined notch (type $\mathrm{v})$, and $b$ and $d$ are the thickness and width, respectively.

The experimental platform for ECPT is shown in Figure 5. It included an infrared camera, an excitation coil, a heating module, and a PC. In the following experiments, the excitation current and frequency were set as $300 \mathrm{~A}$ and $286 \mathrm{kHz}$, respectively. The heating time was set as $200 \mathrm{~ms}$, and the total recording time was $2 \mathrm{~s}$. The FLIR SC650sc IR camera captured thermal images with a spatial resolution of $640 * 120$ pixels at a frequency of $200 \mathrm{~Hz}$, then transmitted the information about the thermal sequences to the computer for later analysis and data postprocessing. The measurement was carried out three times, and the mean was taken.

\section{Results and Discussion}

A typical heating stage experimental result (at $0.2 \mathrm{~s}$ ) for a specimen with a machined V-shaped notch is shown in
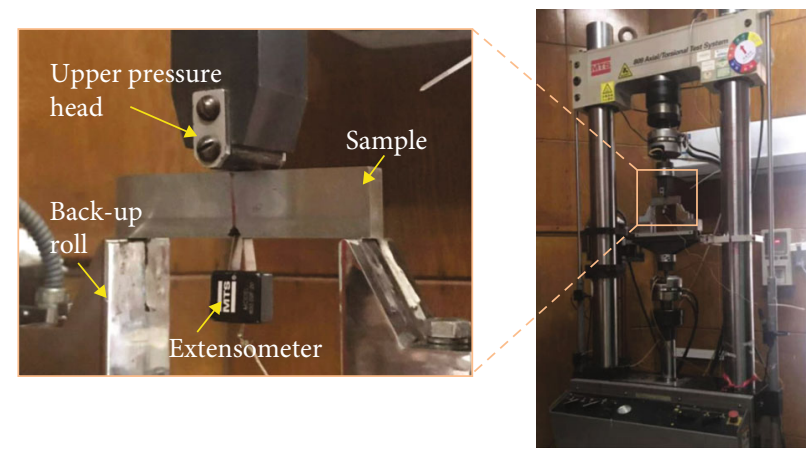

FIgURE 3: Three-point bending test setup.

TABLE 1: Crack length information.

\begin{tabular}{lc}
\hline Specimen no. & Crack length \\
\hline$\# 1$ & $1.0 \mathrm{~mm}$ \\
$\# 2$ & $1.5 \mathrm{~mm}$ \\
$\# 3$ & $2.0 \mathrm{~mm}$ \\
$\# 4$ & $2.5 \mathrm{~mm}$ \\
\hline
\end{tabular}

TABLE 2: Material parameters.

\begin{tabular}{lc}
\hline Parameters & Values \\
\hline Relative permittivity & 100 \\
Conductivity $(\mathrm{S} / \mathrm{m})$ & $5 \times 10^{6}$ \\
Heat capacity $(\mathrm{J} /(\mathrm{kg} \mathrm{K}))$ & 490 \\
Thermal conductivity $(\mathrm{W} /(\mathrm{m} \mathrm{K}))$ & 50 \\
Thermal diffusivity $\left(\mathrm{m}^{2} / \mathrm{s}\right)$ & $1.172 \times 10^{-5}$ \\
Temperature coefficient, $\alpha\left(1 /{ }^{\circ} \mathrm{C}\right)$ & $5.0 \times 10^{-3}$ \\
Density $\left(\mathrm{kg} / \mathrm{m}^{3}\right)$ & $7.7 \times 10^{3}$ \\
Poisson's ratio & 0.3 \\
Elastic modulus $(\mathrm{GPa})$ & 200 \\
\hline
\end{tabular}




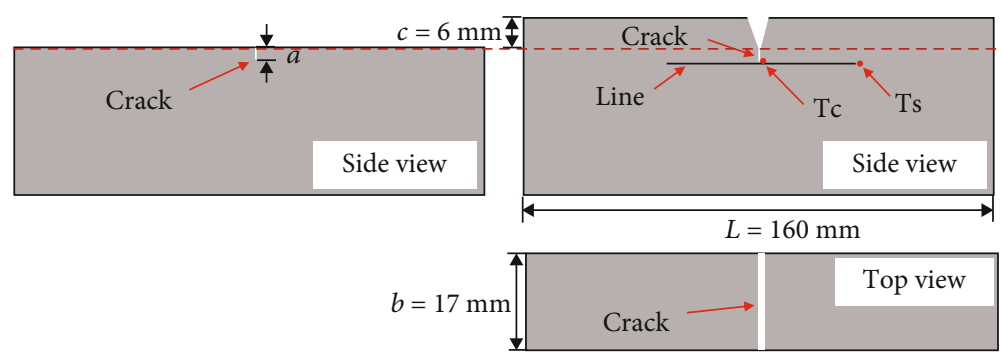

FIgURE 4: Diagram of specimen.

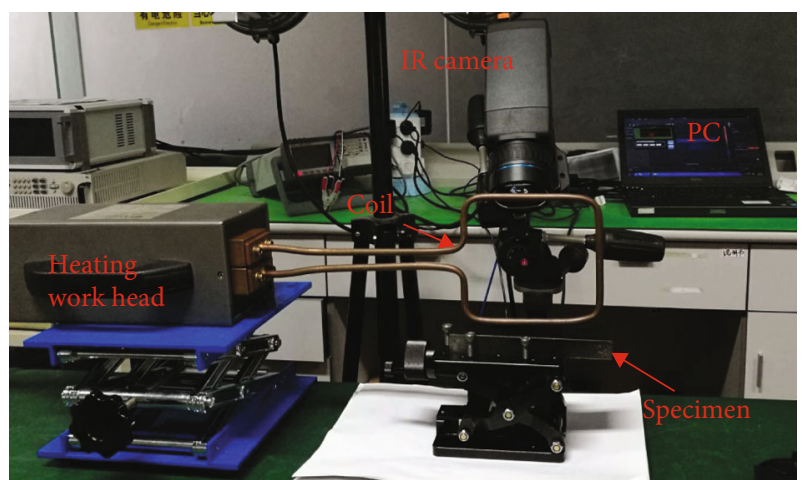

Figure 5: Experimental platform for ECPT.

Figure 6. The region of interest (ROI) including fatigue crack is presented in order to reduce redundant background.

4.1. Temperature Distribution Comparison. The heat distribution on the specimen was uneven due to the defect. In order to investigate the temperature profiles of different types of specimens, a line was created on the surface of specimen \#2 (see Figure 4; specimen after resection should extract data with the same position), and the temperature data on it were extracted (Figure 7). This feature was extracted at the pixel level. From the line scans, Figure 7 shows that (1) temperature presents different distribution in the defective and defect-free areas and (2) the width of the heating up stage becomes narrower when the specimen does not have a V-shaped notch. In detail, these phenomena may be explained by the facts that (1) crack closure effect may be influenced by notches and (2) weak conductivity of closed cracks caused a different temperature response in the medial region because the eddy current distribution was inconsistent between specimens.

Figure 8 illustrates the result of maximum thermal response in the defect region along heating periodicities; the effect of the specimen shape has been investigated. The ratio known as the thermal quotient was used to assess the temperature variation trend, which could be expressed as [27]

$$
T_{q}=\frac{\Delta T_{\text {crack }}}{\Delta T_{\text {surface }}}
$$

Based on the result from Figure 8, it can be seen that both distribution laws are basically equal. The $R$ square was then calculated to evaluate the fitted relation. The specimen with a $\mathrm{V}$-shaped notch has a linear relation with a relatively high $R$ square value (90.15\%). The fluctuations in the unnotched data may be related to crack closure. In addition, the monotonic increase that denotes the final timepoint of heating has a representative defect contrast throughout the whole image sequence.

4.2. Enhanced Feature. A more detailed account of the defect enhancement method is given in this section. Figures 9 and 10 show reconstructed data from 0 to $2 \mathrm{~s}$ using the PCA algorithm for specimen \#4. Setting the same principal components for two different specimens, whether they have a Vshaped notch is the difference between two blocks. To be precise, the first two primary components mostly contained information about the coil and heat diffuses, meaning that the contribution of these two parts played an important role throughout the whole experiment. Meanwhile, component three represents the crack, and the last component denotes the background noise. Clearly, the PCA algorithm enhances defect characteristics; fatigue crack could be detected more easily due to there being less redundant information in the third principal component image.

Figure 11 shows the results processed by the tensor decomposition algorithm at the heating stage (specimen \#4), where the two specimens both contained the low rank (background) part and the sparse (defect) part. From the results, it can be found that the precrack and background are well distinguished. As we mentioned before, background and thermal diffusion occupy the main part of the contribution rate. Therefore, after subtracting the background in the low rank section, the defect morphology is more clearly seen. Red and white boxes indicate typical positions used for SNR analysis. Red ones reflect the defect area while white ones represent the defect-free region.

To verify the effectiveness of the algorithm, eight rail samples with different crack lengths were tested; they ranged in length from $1 \mathrm{~mm}$ to $2.5 \mathrm{~mm}$. Tables 3 and 4 list the results of different signal processing methods for separate trial blocks. In these two tables, component 3 was used as a representative PCA algorithm result. It can be seen in Table 3 that the crack was significantly improved. In Table 4 , four specimens after resection followed a similar pattern, with both PCA and tensor making crack identification easier.

4.3. Performance Evaluation and Comparison. Within our study, the signal-to-noise ratio (SNR) [28] was used to 


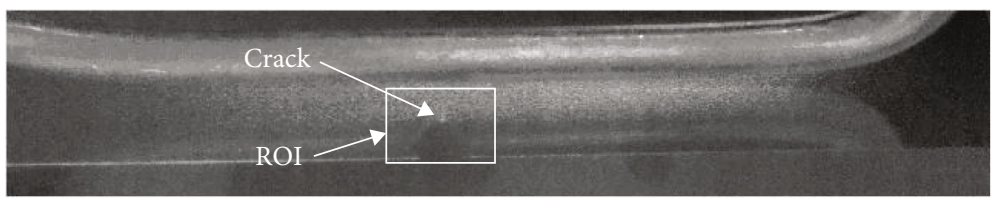

FiguRE 6: Original thermal data and area of interest.

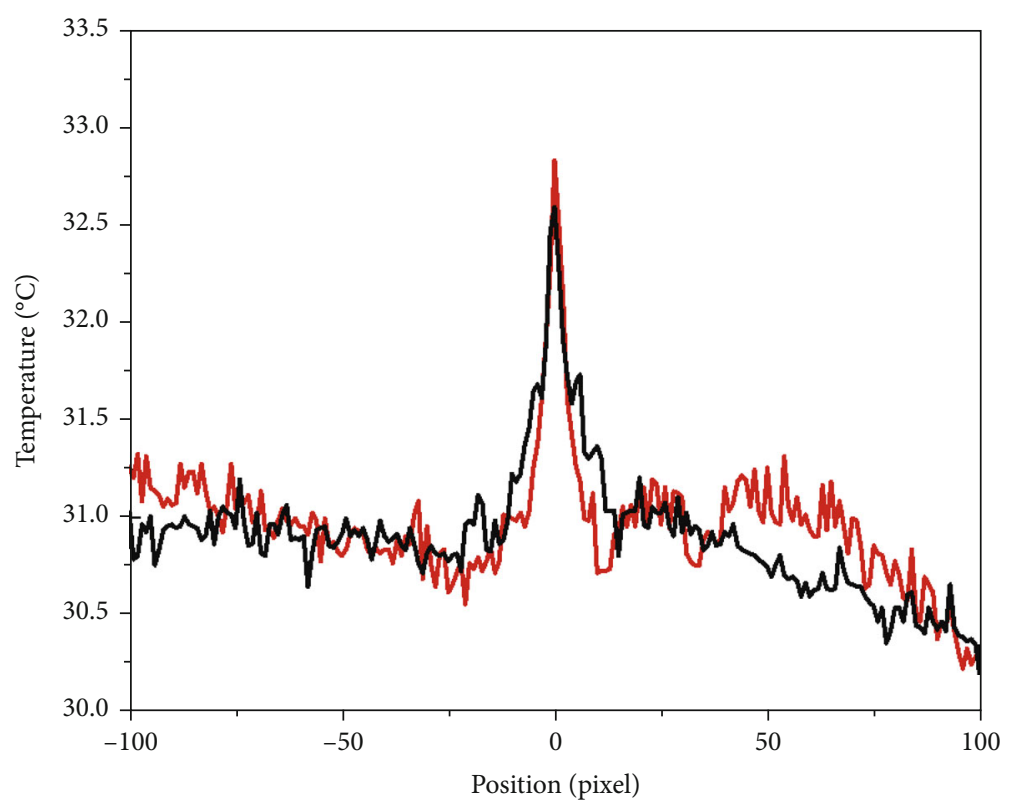

— V-notch specimen with $1.5 \mathrm{~mm}$ crack

_ Specimen without V-notch with $1.5 \mathrm{~mm}$ crack

Figure 7: Temperature line scan of different specimens under $200 \mathrm{~ms}$ heating pulse for specimen \#2.

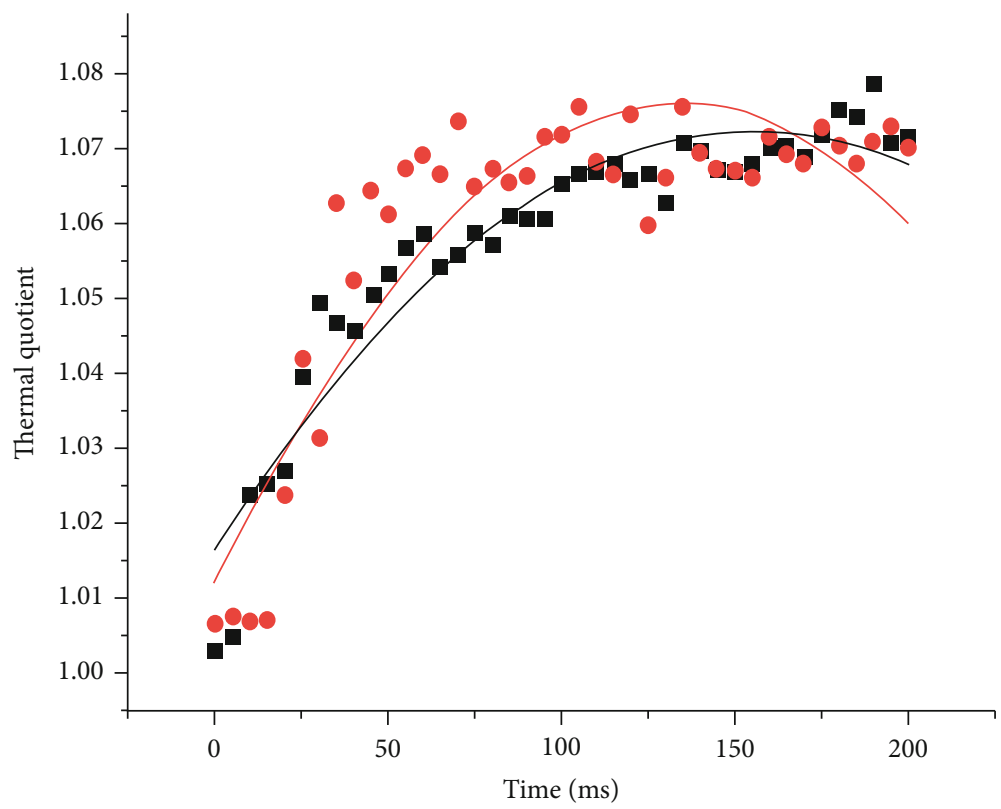

_ Specimen without V-notch with $1.5 \mathrm{~mm}$ crack

_ V-notch specimen with $1.5 \mathrm{~mm}$ crack

Figure 8: Maximum thermal response versus time with second-order polynomial fit of specimen \#2. 

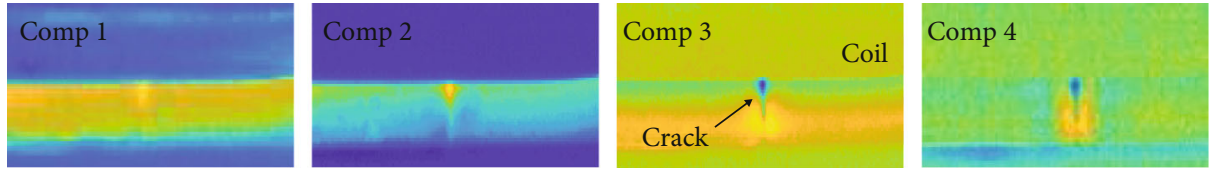

FIgURE 9: PCA results for specimen \#4 without a V-shaped machined notch.
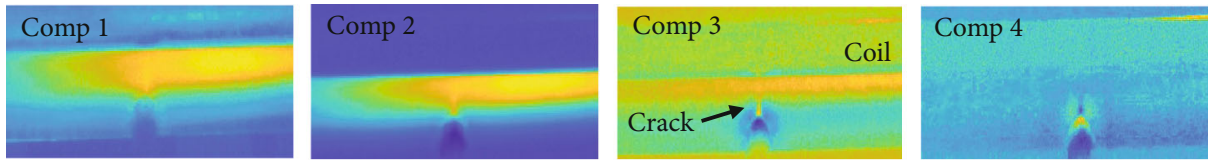

Figure 10: PCA results for notched specimen \#4.

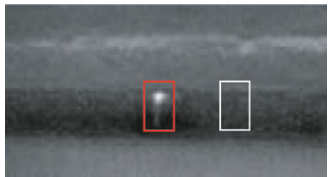

(a)

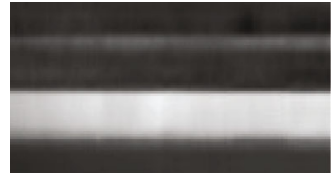

(b)

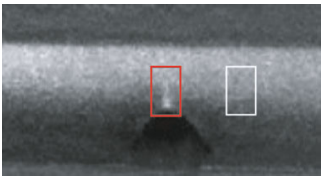

(c)

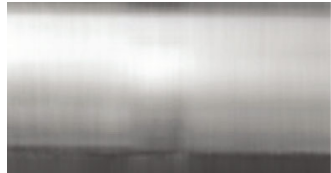

(d)

FIGURE 11: Tensor decomposition results for specimen \#4: (a) sparse rank of sample without notch; (b) low rank of sample without notch; (c) sparse rank of sample with V-shaped notch; (d) low rank of sample with V-shaped notch.

TABLE 3: The results of different signal processing methods for V-notched specimen.

\begin{tabular}{lccc}
\hline & Sample \#1 & Sample \#2 & Sample \#3 \\
\hline Original & & & \\
\hline & & & \\
\hline \\
PCA
\end{tabular}

evaluate the performance of PCA and tensor decomposition. SNR describes the thermal contrast between the defective and nondefective regions; the prefabricated crack region was selected as "signal" and the defect-free region selected as "noise." The calculation of SNR (dB) can be defined using the equation below:

$$
\mathrm{SNR}=20 \log _{10}\left(\frac{\sum_{i=1}^{m} \sum_{j=1}^{n} T_{d(i, j)}}{\sum_{i=1}^{m} \sum_{j=1}^{n} T_{n(i, j)}}\right)
$$

where $\sum_{i=1}^{m} \sum_{j=1}^{n} T_{d(i, j)}$ and $\sum_{i=1}^{m} \sum_{j=1}^{n} T_{n(i, j)}$ are the sum temperature of the crack region and nondefective area, respectively. The original image shows the raw data at $200 \mathrm{~ms}$. The detection performance of various signal processing methods is listed in Table 5; a high SNR value indicates a better crack detection rate. The results show that the Tucker algorithm exhibits a higher crack identification ability than the PCA algorithm in all specimens. Specifically, the result for the original image showed that specimens with a V-shaped notch were more efficient than unnotched samples. This could be 
TABLE 4: The results of different signal processing methods for unnotched specimen.

\begin{tabular}{|c|c|c|c|c|}
\hline & Sample \# 1 & Sample \#2 & Sample \#3 & Sample \#4 \\
\hline Origina & & & & \\
\hline PCA & & & & $=$ \\
\hline Tensor & & & & \\
\hline
\end{tabular}

TABLE 5: The results of the quantitative analysis with SNR in $\mathrm{dB}$.

\begin{tabular}{lccccccc}
\hline & & SNR (with machined notch in dB) & \multicolumn{3}{c}{ SNR (unnotched in dB) } \\
& 1 & 2 & 3 & 4 & 1 & 2 & 3 \\
\hline Original & 2.14 & 3.88 & 3.49 & 3.10 & $\mathrm{X}$ & 2.26 & -0.27 \\
PCA & 2.64 & 4.54 & 6.34 & 4.75 & $\mathrm{X}$ & 2.56 & 2.26 \\
Tucker & 7.18 & 8.18 & 7.65 & 7.30 & $\mathrm{X}$ & 2.80 & 5.05 \\
\hline
\end{tabular}

due to the boundary effect, which generated more heat than in specimens without machined notches. This tendency was also present in the PCA results. In particular, the symbol " $X$ " in the SNR results refers to where the crack could not be detected. Moreover, Table 5 shows that specimens with machined $\mathrm{V}$ notches performed better than unnotched blocks. However, combining crack length and SNR is still controversial; even though it does not affect the robust enhancement algorithm, the relationship is not obvious. This could be due to two causes: the inadequate resolution of the IR camera and the complex internal structure of the crack closure.

\section{Conclusion}

In this paper, eddy current pulsed thermography was investigated to observe fatigue microcracks. Principal component analysis (PCA) and Tucker decomposition were used to extract weak target signals for enhancing detection sensitivity, respectively. Results show that compared with PCA, Tucker decomposition can maintain the structural stability of the original data and extract more crack features that denote a higher SNR. However, it was found that the shape of the sample will influence the detection results. Weak conductivity of closed cracks may change the temperature response in local areas because the crack closure effect may result in localized contact. In addition, the species of closed cracks are not studied in this work, and the linkage between crack length and SNR is not obvious. As such, fur- ther detailed analysis of these factors will be undertaken in future studies.

\section{Data Availability}

The data used to support the findings of this study are available from the corresponding author.

\section{Conflicts of Interest}

The authors declare that they have no conflicts of interest.

\section{Acknowledgments}

This work was supported in part by the National Natural Science Foundation of China under Grant No. 61771409 and the Science and Technology Program of Sichuan under Grant No. 2019YJ0228.

\section{References}

[1] J. Wilson, G. Tian, I. Mukriz, and D. Almond, "PEC thermography for imaging multiple cracks from rolling contact fatigue," NDT \& E International, vol. 44, no. 6, pp. 505-512, 2011.

[2] J. Peng, G. Tian, L. Wang, Y. Zhang, K. Li, and X. Gao, "Investigation into eddy current pulsed thermography for rolling contact fatigue detection and characterization," NDT \& $E$ International, vol. 74, pp. 72-80, 2015. 
[3] Y. He, G. Tian, M. Pan, D. Chen, and H. Zhang, "An investigation into eddy current pulsed thermography for detection of corrosion blister," Corrosion Science, vol. 78, pp. 1-6, 2014.

[4] D. Balageas, X. Maldague, D. Burleigh et al., "Thermal (IR) and other NDT techniques for improved material inspection," Journal of nondestructive evaluation, vol. 35, no. 1, 2016.

[5] X. P. V. Maldague, "Introduction to NDT by active infrared thermography," Materials Evaluation, vol. 60, no. 9, pp. 1060-1073, 2002.

[6] H. Zhang, S. Sfarra, F. Sarasini et al., "Optical and mechanical excitation thermography for impact response in basalt-carbon hybrid fiber-reinforced composite laminates," IEEE Transactions on Industrial Informatics, vol. 14, no. 2, pp. 514-522, 2018.

[7] J. Vrana, M. Goldammer, J. Baumann, M. Rothenfusser, and W. Arnold, "Mechanisms and models for crack detection with induction thermography," AIP Conference Proceedings, vol. 975, no. 1, pp. 475-482, 2008.

[8] Z. Shi, X. Xu, J. Ma, D. Zhen, and H. Zhang, "Quantitative detection of cracks in steel using eddy current pulsed thermography," Sensors, vol. 18, no. 4, 2018.

[9] B. Weekes, D. P. Almond, P. Cawley, and T. Barden, "Eddycurrent induced thermography-probability of detection study of small fatigue cracks in steel, titanium and nickel-based superalloy," NDT \& E International, vol. 49, pp. 47-56, 2012.

[10] Z. Tong, S. Xie, H. Liu et al., "An efficient electromagnetic and thermal modelling of eddy current pulsed thermography for quantitative evaluation of blade fatigue cracks in heavy-duty gas turbines," Mechanical Systems and Signal Processing, vol. 142, p. 106781, 2020.

[11] N. Udo, G. Walle, S. Lugin, A. Ehlen, S. Bessert, and B. Valeske, "Induction thermography: principle, applications and first steps towards standardisation," Quantitative InfraRed Thermography Journal, vol. 13, pp. 170-181, 2016.

[12] P. Yu, S. Huang, Y. He, and X. Guo, "Eddy current pulsed thermography for noncontact nondestructive inspection of motor winding defects," IEEE Sensors Journal, vol. 20, no. 5, pp. 2625-2634, 2020.

[13] M. Genest and G. Li, "Induction thermography of steel coupons with cracks," Applied Optics, vol. 57, no. 18, pp. d40$\mathrm{d} 48,2018$.

[14] X. Maldague and S. Marinetti, "Pulse phase infrared thermography," Journal of Applied Physics, vol. 79, no. 5, pp. 26942698, 1996.

[15] K. Chen, L. Bai, Y. Chen, Y. Cheng, S. Tian, and P. Zhu, "Defect automatic identification of eddy current pulsed thermography," Journal of Sensors, vol. 2014, Article ID 326316, 7 pages, 2014.

[16] Y. Wang, B. Gao, W. L. Woo et al., "Thermal pattern contrast diagnostic of microcracks with induction thermography for aircraft braking components," IEEE Transactions on Industrial Informatics, vol. 14, no. 12, pp. 5563-5574, 2018.

[17] H. Zhang, S. Sfarra, A. Osman et al., "Eddy current pulsed thermography for ballistic impact evaluation in basalt-carbon hybrid composite panels," Applied Optics, vol. 57, no. 18, pp. 74-81, 2018.

[18] B. Gao, Y. He, W. L. Woo, G. Y. Tian, J. Liu, and Y. Hu, "Multidimensional tensor-based inductive thermography with multiple physical fields for offshore wind turbine gear inspection," IEEE Transactions on Industrial Electronics, vol. 63, no. 10, pp. 6305-6315, 2016.
[19] J. Song, B. Gao, W. L. Woo, and G. Y. Tian, "Ensemble tensor decomposition for infrared thermography cracks detection system," Infrared Physics \& Technology, vol. 105, p. 103203, 2020.

[20] G. Lesiuk, M. Szata, J. A. F. O. Correia, A. M. P. De Jesus, and F. Berto, "Kinetics of fatigue crack growth and crack closure effect in long term operating steel manufactured at the turn of the $19^{\text {th }}$ and $20^{\text {th }}$ centuries," Engineering Fracture Mechanics, vol. 185, pp. 160-174, 2017.

[21] J. A. F. O. Correia, S. Blasón, and A. Arcari, "Modified CCS fatigue crack growth model for the AA2019-T851 based on plasticity-induced crack-closure," Theoretical and Applied Fracture Mechanics, vol. 85, pp. 26-36, 2016.

[22] G. T. Gray, J. C. Williams, and A. W. Tompson, "Roughnessinduced crack closure: an explanation for microstructurally sensitive fatigue crack growth," Metallurgical Transactions A, vol. 14, no. 2, pp. 421-433, 1983.

[23] K. Asami and H. Emura, "The influence of moisture in air on fatigue crack propagation characteristics of high-strength steels," Journal of the Society of Materials Science, vol. 39, no. 439, pp. 425-431, 1990.

[24] K. Tokaji, Z. Ando, and K. Nagae, "The effect of sheet thickness on near-threshold fatigue crack propagation and oxide and roughness-induced crack closure," Journal of Engineering Materials and Technology, vol. 109, no. 1, pp. 86-91, 1987.

[25] C. Jomdecha, W. Cai, S. Xie, Y. Li, and Z. Chen, "Analysis of magnetic flux perturbation due to conductivity variation in equivalent stress-corrosion crack," International Journal of Applied Electromagnetics and Mechanics, vol. 59, no. 4, pp. 1385-1392, 2019.

[26] Z. Chen, Z. Chen, N. Yusa, and K. Miya, "A nondestructive strategy for the distinction of natural fatigue and stress corrosion cracks based on signals from eddy current testing," Journal of Pressure Vessel Technology, vol. 129, no. 4, pp. 719-728, 2007.

[27] B. Oswald-Tranta, "Thermo-inductive crack detection," Nondestructive Testing and Evaluation, vol. 22, no. 2-3, pp. 137-153, 2007.

[28] F. Lopez, C. Ibarra-Castanedo, V. de Paulo Nicolau, and $\mathrm{X}$. Maldague, "Optimization of pulsed thermography inspection by partial least-squares regression," NDT \& E International, vol. 66, pp. 128-138, 2014. 